\title{
Prévalence de la gale sarcoptique chez le porc dans le département de la Mifi (Ouest Cameroun)
}

\author{
Alain Kouam Simo ${ }^{1 *}$ Félicité Flore Djuikwo-Teukeng ${ }^{1}$ \\ Christian Kombou Fangye ${ }^{1}$ Guy-Pierre Martineau ${ }^{2}$ \\ Mohamed Gharbi ${ }^{3}$ Philippe Dorchies ${ }^{2}$
}

\section{Mots-clés}

Porcin, Sarcoptes scabiei var. suis, gale, Cameroun

\author{
Submitted: 17 May 2019 \\ Accepted: 18 August 2020 \\ Published: 1 December 2020 \\ DOI: $10.19182 /$ remvt.31943
}

\begin{abstract}
Résumé
La gale du porc, due à Sarcoptes scabiei var. suis, est l'une des principales dermatoses porcines, présente dans de nombreux élevages. Elle provoque d'importantes pertes économiques dans plusieurs pays et son éradication passe par une amélioration des conditions d'hygiène. En l'absence de données récentes sur la prévalence de cette infestation au Cameroun, la recherche des parasites par raclages cutanés a été réalisée dans 52 élevages de la région de l'Ouest (département de la Mifi). En avril-août 2015, puis en avril-août 2016, 359 raclages cutanés sur 103 truies, 39 verrats et 217 porcelets sevrés ont permis d'identifier S. scabiei var. suis dans $23,1 \%$ des élevages (12/52; soit 40 porcheries exemptes de gale) avec une prévalence moyenne de 19,5\% (70/359), sans différence de sexe ni d'âge. Les porcheries les plus infestées étaient celles où l'hygiène était la plus défectueuse. Des programmes d'éducation sanitaire doivent être mis en place pour sensibiliser les éleveurs à l'importance du respect des règles d'hygiène dans la lutte contre la gale porcine.
\end{abstract}

- Comment citer cet article : Kouam Simo A., Djuikwo-Teukeng F.F., Kombou Fangye C., Martineau G.-P., Gharbi M., Dorchies P., 2020. Prevalence of sarcoptic mange in pigs in Mifi Department (West Cameroon). Rev. Elev. Med. Vet. Pays Trop., 73 (4): 269-271, doi: 10.19182/remvt.31943

\section{INTRODUCTION}

La gale sarcoptique du porc (due à Sarcoptes scabiei var. suis) est fréquente dans de nombreux pays du monde. Elle est souvent difficile à éradiquer si des mesures sévères de biosécurité, associées à l'utilisation d'acaricides de bonne qualité employés aux posologies ou concentrations recommandées, ne sont pas assorties d'une désinfection des locaux d'élevage (Dagleish et al., 2007 ; Walton et Currie, 2007 ; Eo et al., 2008 ; Chhabra et Pathak, 2011). La gale se traduit, deux à trois semaines après la contamination de l'animal, par l'apparition de taches érythémateuses de 2-3 millimètres de diamètre sur le corps (Martineau et al., 1987), suivie de la formation de croûtes, d'une hyperkératose et de la chute des soies, laissant une peau épaissie et rugueuse (Das et al., 2010).

Les enquêtes réalisées dans divers pays afin de déterminer la prévalence de cette maladie utilisent soit le dosage immunoenzymatique (Elisa), soit les raclages cutanés. Les premiers montrent la persistance et la circulation des parasites même en l'absence de symptômes (par exemple $25 \%$ des troupeaux et $2 \%$ des truies infestées de gale au

\footnotetext{
1. Université des Montagnes, Faculté des sciences de la santé, PO Box 208, Bangangté, Cameroun.

2. Ecole nationale vétérinaire de Toulouse, 31076 Toulouse, France.

3. Laboratoire de parasitologie, Université Manouba, Ecole nationale de médecine vétérinaire de Sidi Thabet, Sidi Thabet, Tunisie.

* Auteur pour la correspondance

Email : alkouam2005@yahoo.fr
}

Québec ; Ravel et al., 2002). Les autres études permettent la mise en évidence des parasites, attestant ainsi de l'actualité de l'infestation. Au nord de l'Inde, une enquête réalisée sur 196 porcs a révélé que $11 \%$ d'entre eux étaient galeux après un examen des croûtes (Laha et al., 2014). Dans 110 élevages de Hesse, en Allemagne, 19,1\% des 2754 truies examinées étaient atteintes de gale sarcoptique. La perte économique consécutive a été évaluée à 4200 euros par exploitation et par an dans 850 élevages contenant au total 17000 truies (Damriyasa et al., 2004). Au Québec, les pertes dues à la gale ont été estimées entre 84 et 115 \$US par truie infestée par an, essentiellement à cause d'une diminution des performances à l'engraissement (Ravel et al., 2002).

La gale induit des pertes économiques qu'il est cependant parfois difficile de quantifier. Elles sont notamment liées aux baisses de production consécutives à l'hyporexie liée au surmenage induit par le prurit quasi permanent et à la dépréciation des carcasses en relation avec les lésions cutanées obligeant dans certains cas à effectuer le parage desdites carcasses à l'abattoir (Gérôme, 2008 ; OIE, 2008). La gale sarcoptique serait à l'origine d'une diminution de la vitesse de croissance de $10 \%$ en moyenne (Cargill et Dobson, 1979).

Au Cameroun, l'élevage du porc s'est développé à partir des années 1980 dans les régions de l'Ouest, du Littoral et du Centre. Cette production est sans cesse croissante, en particulier dans la région de l'Ouest où les conditions d'élevage sont favorables au développement de l'agriculture (INSC, 2015). Le manque de données épidémiologiques actualisées concernant la gale porcine a justifié cette étude qui a eu pour objectif d'estimer la prévalence de la gale sarcoptique porcine dans le département de la Mifi. 


\section{MATERIEL ET METHODES}

\section{Région d'étude et élevages}

Le département de la Mifi est situé à l'ouest du Cameroun ( $5^{\circ} 30^{\prime} \mathrm{N}$; $10^{\circ} 23^{\prime}$ E). Il se caractérise par un climat doux, de type tropical à faciès montagnard. La température annuelle moyenne est de $25^{\circ} \mathrm{C}$, avec deux saisons des pluies : la petite (de mars à mai) et la grande (de juin à septembre).La pluviométrie annuelle moyenne est de 916 millimètres (Météo Mifi). L'étude s'est déroulée sur deux périodes de cinq mois correspondant à la saison des pluies (avril-août 2015 puis avril-août 2016). Les élevages étaient indépendants les uns des autres durant toute la période de prélèvement. Cinquante-deux élevages ont été inclus dans l'étude au cours de laquelle des prélèvements ont été réalisés sur 359 porcs de diverses races (Large White, Duroc, Piétrain et croisés). Le niveau de propreté des élevages a été évalué. Ils ont été répartis en trois catégories : les élevages propres (nettoyages réguliers, quotidiens), les élevages moyennement propres (nettoyages une fois tous les deux jours), et les élevages sales (nettoyages hebdomadaires) où les fèces s'accumulaient, les mangeoires et les abreuvoirs étaient sales et encombrés de détritus.

\section{Examen des animaux et des échantillons}

Lors de l'arrivée dans chaque porcherie, le même observateur s'installait dans une partie élevée pour voir facilement le maximum de porcs. Il restait alors immobile pendant quelques minutes afin que les porcs se calment et reprennent leurs comportements habituels. Ensuite, les porcs de chaque loge ont été observés pendant 15 minutes. Le nombre d'animaux qui se grattaient et l'intensité du prurit (faible, moyen et intense) étaient relevés.

Des raclages cutanés ont ensuite été effectués sur sept animaux en moyenne par élevage. Les animaux prélevés étaient soit ceux suspects de gale (donc présentant soit du prurit, soit des croûtes, soit des dépilations, voire deux ou trois signes), soit les animaux apparemment sains présents dans une loge d'un animal suspect. Aucun prélèvement n'a été effectué à partir des conduits auditifs des animaux car aucune lésion auriculaire n’a été constatée.

Les raclages cutanés ont été conservés dans l'alcool à $60 \%$ puis dissous dans une solution d'hydroxyde de potassium à $10 \%$ dans un bain-marie en ébullition pendant $10 \mathrm{~min}$. La suspension a ensuite été centrifugée à 3000 tours/min pendant $3 \mathrm{~min}$. Le culot a été examiné sous une loupe binoculaire au grossissement x10 pour l'identification des sarcoptes (OIE, 2008).

\section{Analyses statistiques}

Les prévalences d'infections ont été comparées avec le test de chideux au risque de $5 \%$ (Schwartz, 1993).

\section{- RESULTATS ET DISCUSSION}

Dans la Mifi, $80 \%$ des éleveurs de porcs sont des naisseurs-engraisseurs, $10 \%$ sont des engraisseurs et autant sont des naisseurs. Les porcelets sont vendus au sevrage, entre 45 jours et deux mois d'âge. Les porcs sont maintenus en claustration permanente. Les installations des fermes sont diverses. Certaines ont des loges avec un sol cimenté et des cloisons en planches (semi-dur), d'autres possèdent des loges en bois montées sur pilotis, d'autres sont en dur (sols cimentés et cloisons en parpaings) et quelques-unes sont en bambous.

Lors de cette étude, 23,1 \% (12/52) des élevages ont été observés atteints de gale. C'était aussi le cas pour 6/21 (29\%) des porcheries sur pilotis, $5 / 20(25 \%)$ des installations en semi-dur et $1 / 3(33 \%)$ de celles en bambou. Par contre, aucun des huit élevages construits en dur n'abritait d'animaux infestés. Tous les élevages cliniquement suspects de gale ont été confirmés positifs au laboratoire : ils hébergeaient au moins un animal infesté par Sarcoptes scabiei.
La proportion de raclages cutanés positifs (présentant au moins un Sarcoptes) était de 19,5\% (70/359). La prévalence estimée dans la présente étude était inférieure à celle rapportée par Alonso de Vega et al. (1998) au sud-est de l'Espagne (37\%) mais supérieure à celle de $10 \%$ observée au nord-est de l'Inde (Laha et al., 2014). La prévalence d'infestation était comparable au sein des différentes catégories d'âges (tableau I). Elle était de 19,4\% (42/217) chez les porcelets, $20,4 \%(21 / 103)$ chez les truies et $17,9 \%$ (7/39) chez les verrats $(\mathrm{p}=0,7)$. Il n'y avait donc aucune différence entre les sexes et les âges dans la population échantillonnée.

Dans cette enquête, 17 \% (61/359) des porcs examinés avaient un prurit mais environ un tiers d'entre eux $(33,4 \% ; 120 / 359)$ présentaient de l'hyperkératose sur les flancs et le cou. Ces lésions cutanées pouvaient avoir parmi trois origines : a) une gale traitée récemment et en voie de guérison ; b) des lésions en relation avec de l'hypersensibilité consécutive aux réinfestations fréquentes d'animaux immunisés; et c) l'existence d'une parakératose d'origine nutritionnelle chez ces animaux recevant des régimes alimentaires déséquilibrés à base de son de blé et de restes de cuisine. Parmi les sujets présentant des lésions de prurit, presque la moitié $(42,6 \% ; 26 / 61)$ exprimait un prurit quasi permanent alors que les autres ne se grattaient qu'une à deux fois par 15 minutes. De plus, l'examen clinique des porcs a montré que $20 \%$ (72/359) d'entre eux présentaient des dépilations plus ou moins étendues sur les flancs et la ligne du dos.

Au total, 31,8 \% (61/192) des porcs ayant du prurit, de l'hyperkératose ou des dépilations étaient porteurs de $S$. scabiei. La prévalence du portage asymptomatique de $S$. scabiei était de 2,5\% (9/359) parmi les animaux examinés. La présence d'animaux infestés par des sarcoptes mais n'ayant aucun symptôme pouvait s'expliquer par le fait que les éleveurs appliquaient parfois de l'huile de vidange sur les animaux pour traiter ceux qui avaient un prurit. Cette huile servait aussi d'émollient et à éliminer les croûtes. La prévalence d'infestation était différente en fonction du niveau d'hygiène des bâtiments $(\mathrm{p}=0,01)$ (tableau I).

\section{CONCLUSION}

L'étude menée dans 52 élevages de porcs du département de la Mifi (Ouest Cameroun) a permis d'estimer la prévalence de la gale

Tableau I : Prévalence de l'infection de porcins par Sarcoptes scabiei en fonction de certains facteurs de risque (Ouest Cameroun) I/I Prevalence of infection of pigs by Sarcoptes scabiei according to some risk factors (West Cameroon)

\begin{tabular}{lcc} 
Facteur de risque & Positifs/examinés $(\%)$ & p \\
\hline Animaux & & \\
Verrats & $7 / 39(17,9)$ & \\
Truies & $21 / 103(20,4)$ & \\
Porcelets & $42 / 217(19,4)$ & \\
Total & $70 / 359(19,5)$ & 0,7 \\
Type de local d'élevage & & \\
Pilotis & $6 / 21(28,6)$ & \\
Semi-dur & $5 / 20(25,0)$ & \\
Dur & $0 / 8(0,0)$ & \\
Bambou & $1 / 3(33,3)$ & \\
Total & $12 / 52(23,1)$ & 0,39 \\
Etat d'hygiène du local d'élevage & & \\
Propre & $0 / 17(0,0)$ & \\
Moyennement propre & $4 / 16(25,0)$ & \\
Sale & $8 / 19(42,1)$ & \\
Total & $12 / 52(23,1)$ & 0,01
\end{tabular}


sarcoptique des porcs à 19,5\%. Le niveau d'hygiène des bâtiments a eu un impact sur la présence de ces parasites, aucun des 17 élevages classés comme propres n'ayant abrité d'animaux infestés. La gale sarcoptique était encore largement répandue dans les élevages de porcs de la région d'étude. Les éleveurs devraient renforcer l'hygiène pour lutter de manière efficace contre $S$. scabiei et donc la santé des porcs dans la Mifi.

\section{Remerciements}

Les auteurs remercient tous les éleveurs de porcs qui les ont autorisés à accéder à leurs élevages ainsi que les responsables du ministère de l'Elevage du Cameroun qui les ont autorisés à contacter les éleveurs.

\section{Déclaration des contributions des auteurs}

$\mathrm{PD}, \mathrm{FFDT}$ et $\mathrm{AKS}$ ont participé à la conception et à la planification de l'étude ; CKF et AKS ont recueilli les données sur le terrain ; AKS et FFDT ont rédigé la première version du manuscrit ; PD, GPM et MG ont révisé le manuscrit.

\section{Conflits d'intérêts}

Les auteurs déclarent que l'étude a été réalisée sans conflit d'intérêts.

\section{REFERENCES}

Alonso de Vega F., Mendez de Vigo J., Ortiz Sanchez J., Martinez-Carrasco Pleite C., Albaladejo Serrano A., Ruiz de Ybañez Carnero M.R., 1998. Evaluation of the prevalence of sarcoptic mange in slaughtered fattening pigs in southeastern Spain. Vet. Parasitol., 76 (3): 203-209, doi : 10.1016/ S0304-4017(97)00212-4

Cargill C.F., Dobson K.J., 1979. Experimental Sarcoptes scabiei infestation in pigs: (2) Effects on production. Vet. Rec., 104 (2): 33-36, doi: 10.1136/vr.104.2.33

Chhabra M., Pathak K.M.L., 2011. Sarcoptic mange in domestic animals and human scabies in India. J. Vet. Parasitol., 25 (1): 1-10

Dagleish M.P., Ali Q., Powell R.K., Butz D., Woodford M.H., 2007. Fatal Sarcoptes scabiei infection of blue sheep (Pseudois nayaur) in Pakistan. J. Wildl. Dis., 43 (3): 512-517, doi: 10.7589/0090-3558-43.3.512

\section{Summary}

Kouam Simo A., Djuikwo-Teukeng F.F., Kombou Fangye C., Martineau G.-P., Gharbi M., Dorchies P. Prevalence of sarcoptic mange in pigs in Mifi Department (West Cameroon)

Pig scabies, caused by Sarcoptes scabiei var. suis, is one of the main pig dermatoses and is frequently present in pig farms. It causes important economic losses in several countries and its eradication involves improving hygienic conditions. In the absence of recent data on the prevalence of this infestation in Cameroon, the search for parasites by skin scraping was carried out in 52 farms in the Western region (Mifi Department). In April-August 2015, then in April-August 2016, 359 skin scrapings on 103 sows, 39 boars and 217 weaned piglets allowed to identify S. scabiei var. suis in $23.1 \%$ of the farms (12/52; i.e. 40 scabies-free pig farms). The mean prevalence was $19.5 \%$ (70/359) with no difference in regard to sex or age. The most infested pigsties were those where hygiene was the poorest. Health education programs need to be implemented to sensitize farmers to the importance of hygiene compliance in order to control swine scabies.

Keywords: swine, Sarcoptes scabiei var. suis, mange, Cameroon
Damriyasa I.M., Failing K., Volmer R., Zahner H., Bauer C., 2004. Prevalence, risk factors and economic importance of infestations with Sarcoptes scabiei and Haematopinus suis in sows of pig breeding farms in Hesse, Germany. Med. Vet. Entomol., 18 (4): 361-367, doi: 10.1111/j.0269-283X.2004.00520.x

Das M., Laha R., Devi P., Bordoloi R.K., Naskar S., 2010. Sarcoptic mange infestation in pigs in a hilly region of Meghalaya. Trop. Anim. Health Prod., 42 (5): 1009-1011, doi: 10.1007/s11250-009-9523-4

Eo K.Y., Kwon O.D., Shin N.S., Shin T., Kwak D., 2008. Sarcoptic mange in wild raccoon dogs (Nyctereutes procyonoides) in Korea. J. Zoo Wildl. Med., 39 (4): 671-673, doi: 10.1638/2008-0034.1

Gérôme S., 2008. Analyse des impacts de la décharge de Mbeubeuss sur les élevages porcins environnants. Thèse Doct., Université Cheikh Anta Diop, Dakar, Sénégal, 108 p.

INSC (Institut National de la Statistique du Cameroun), 2015. Annuaire Statistique du Cameroun. Chapitre 15 : Élevage et Pêche. INSC, Yaoundé, Cameroun, www.stat.cm/downloads/2016/annuaire2016/CHAPITRE15_PECHE_ ELEVAGE.pdf (consulté le 28/09/2019)

Laha R., Das M., Bharti P.K.., Kumar S., Sen A., Goswami A., 2014. Prevalence of Sarcoptes scabiei var. suis infestation in pigs of Meghalaya and its treatment. Vet. World, 7 (12): 1137-1139, doi: https://doi.org/10.14202/ vetworld.2014.1137-1139

Martineau G.P., van Neste D., Charette R., 1987. Pathophysiology of sarcoptic mange in swine. Comp. Contin. Educ. Pract. Vet., 9 (2), F51-F58

Météo Mifi, 2019. Données disponibles sur planificateur.a-contresens.net/afrique/cameroun/ouest/mifi/9085341.html (consulté le 29/06/2019)

OIE, 2008. Manuel des tests de diagnostic et des vaccins pour les animaux terrestres (mammifères, oiseaux et abeilles). Chapitre 2.9.8. Gales, 13731386, https://studylibfr.com/doc/6456813/organisation-mondiale-de-la-santé-animale (consulté le 19/07/2019)

Ravel A., Chamberlin J., Cloutier S. 2002. La gale dans les troupeaux de truies au Québec. Porcs Québec, 5p. http://www.agrireseau.net/porc/Documents/recherche-gale-m.pdf (consulté le 05/09/2019)

Schwartz D, 1993. Méthodes statistiques à l'usage des médecins et des biologistes, $4^{\text {ème }}$ Ed. Flammarion, Paris, France, 315 p.

Walton S.F., Currie B.J. 2007. Problems in diagnosing scabies, a global disease in human and animal populations. Clin. Microbiol. Rev., 20 (2): 268-279, doi: h10.1128/CMR.00042-06

\section{Resumen}

Kouam Simo A., Djuikwo-Teukeng F.F., Kombou Fangye C., Martineau G.-P., Gharbi M., Dorchies P. Prevalencia de la sarna sarcoptica en cerdos en el departamento de Mifi (Camerún del Oeste)

La sarna porcina, causada por Sarcoptes scabiei var. suis, es una de las principales dermatitis en cerdos y se encuentra frecuentemente en las fincas de cerdos. Provoca importantes pérdidas económicas en varios países y su erradicación incluye mejoramiento de las condiciones higiénicas. En vista de la ausencia de datos recientes sobre la prevalencia de esta infestación en Camerún, se llevó a cabo una búsqueda de parásitos mediante raspados de piel en 52 fincas, en la región Oeste (departamento de Mifi). Entre abril-agosto 2015, y luego abril-agosto 2016, 359 raspados en 103 hembras, 39 machos y 217 lechones destetados permitieron la identificación de $S$. scabiei var. suis en $23,1 \%$ de las fincas $(12 / 52 ; 40$ fincas estuvieron libres de sarna). La prevalencia promedio fue de 19,5\% (70/359) sin diferencia con respecto al sexo o la edad. Las porquerizas con mayor infestación fueron aquellas con la menor higiene. Programas de educación sanitaria deben implementarse para sensibilizar a los finqueros sobre la importancia del respeto de la higiene, con el fin de controlar la sarna porcina.

Palabras clave: porcino, Sarcoptes scabiei var. suis, Sarna, Camerún 
